\title{
Modelling and Simulation of Wildland Fire in the Framework of the Level Set Method
}

\author{
Andrea Mentrelli • Gianni Pagnini
}

Received: date / Accepted: date

\begin{abstract}
Among the modelling approaches that have been proposed for the simulation of wildfire propagation, two have gained considerable attention in recent years: the one based on a reaction-diffusion equation, and the one based on the level set method. These two approaches, traditionally seen in competition, do actually lead to similar equation models when the level set method is modified taking into account random effects as those due to turbulent hot air transport and fire spotting phenomena.

The connection between these two approaches is here discussed and the application of the modified level set method to test cases of practical interest is shown.
\end{abstract}

Keywords Wildland Fire Simulation · Level Set Method · Reaction-Diffusion Model

\section{Introduction}

In recent years wildfire propagation modelling and simulation has drawn considerable attention and many efforts in this challenging research field have been made, as thoroughly reviewed in [1-3]. Among the others, two different approaches have proven to be successful: one is based on a reaction-diffusion model [4-6], and the other one on the so-called level set method (LSM) [7-9].

These two approaches are typically regarded as mutually exclusive, being the solution of a reaction-diffusion equation a smooth function representing, for instance, the temperature in the domain of interest, and the solution of the LSM providing the

Andrea Mentrelli

Department of Mathematics \& Alma Mater Research Center on Applied Mathematics $\left(\mathrm{AM}^{2}\right)$

University of Bologna - Bologna, Italy

and Basque Center for Applied Mathematics (BCAM) - Bilbao, Basque Country, Spain

E-mail: andrea.mentrelli@unibo.it

Gianni Pagnini

Basque Center for Applied Mathematics (BCAM) - Bilbao, Basque Country, Spain

and Ikerbasque, Basque Foundation for Science - Bilbao, Basque Country, Spain

E-mail: gpagnini@bcamath.org 
exact localization of the fire front. However, it was recently argued that these two approaches are complementary and can be merged $[10,11]$.

Two features of wildfire propagation which are remarkably challenging from the modelling point of view are the turbulent transport phenomena in hot regions [12-15], and the fire spotting, i.e. the ejection of embers from the hot region into the yet-toburn region ahead of the fire [16-18]. Since both these processes at a macroscopic level have random characters, the fire evolution turns out to be a stochastic process [19], and the level set method has proven to be a flexible framework that allows to implement these random phenomena with relative ease.

In the model recently adopted and improved by the authors [20-22], the fire front motion is randomized by adding to the deterministic rate of spread $(\mathrm{RoS})$ of the fire prescribed by empirical or semi-empirical models, two stochastic terms corresponding to the above mentioned turbulent heat transport and fire spotting. The resulting averaged process is governed by an evolution equation of reaction-diffusion type in which the rate of spread (RoS) of the fire plays a role in the source term and the randomization of the fire front is performed according to the probability density function (PDF) of the front particle displacement. In the limit case of deterministic motion, the reaction-diffusion equation reduces to the Hamilton-Jacobi equation typical of the classical LSM. The resulting model allows to simulate the effects of fire-breaks something that cannot be easily modelled by the classical level set method - as well as effects of turbulent convection such as flanking and backing fire and the increase in fire spread due to the pre-heating associated to turbulent transport of hot air.

The paper is organized as follows. In Section 2 the models of wildfire propagation based on the reaction-diffusion equation (Section 2.1) and on the LSM (Section 2.2) are outlined, as well as the newly introduced randomized LSM (Section 2.3). In Section 3 the application of the proposed model is discussedand in Section 4 a selection of numerical simulation results are shown and discussed.

\section{Reaction-Diffusion Model and Level Set Method in Wildfire Simulation}

\subsection{Brief overview of the rection-diffusion model}

An important observable in regions affected by fire is the temperature. Since the mechanisms of energy transport involve molecular processes in turbulent flows, on a macroscopic level the transport of energy can be modelled as a diffusion process and the combustion is modelled as a source term. A two-equation model involving the average temperature field $T(\mathbf{x}, t)$ and the fuel mass fraction $Y(\mathbf{x}, t), Y \in[0,1]$, has been developed and has proven to be very successful $[4-6,23,24]$. In its simpler formulation, such a model is based on the following governing equations

$$
\frac{\partial T}{\partial t}+\mathbf{U} \nabla T=\nabla(K \nabla T)+R Y-\left(T-T_{a}\right), \quad \frac{\partial Y}{\partial t}=-R Y,
$$

where $\mathbf{U}$ is the mean wind velocity, $K$ the diffusion coefficient, $R$ the reaction rate and $T_{a}$ the ambient temperature. 
In models based on a reaction-diffusion equation, typically a solution featuring large gradients in the neighbourhood of the fire front, and nearly constant elsewhere, as to allow to localize of the fire front, is regarded as an important asset. This consideration leads directly to the development of a model based on the LSM. The latter, in fact, provides a well-known and flexible framework for modelling the evolution of moving interfaces [7]. In the context of wildfire propagation, modelling the interface between burnt/unburnt regions by means of the classical LSM has been considered by several authors $[8,9,13]$.

\subsection{The classical level set method}

The level set method can be briefly described as follows [7]. Let $\Gamma \equiv \Gamma(t)$ be the interface (either a simple closed curve, or an ensemble of simple non-intersecting closed curves), representing a propagating front in a domain $\mathcal{S} \subseteq \mathbb{R}^{d}(d=2,3)$, and let $\varphi: \mathcal{S} \times\left[0,+\infty\left[\rightarrow \mathbb{R}\right.\right.$ be a function such that $\varphi(\mathbf{x}, t)=\varphi_{*}$ coincides with the evolving front, i.e. $\Gamma(t)=\left\{\mathbf{x} \in \mathcal{S} \mid \varphi(\mathbf{x}, t)=\varphi_{*}\right\}$. The evolution of the field $\varphi$ is governed by the following Hamilton-Jacobi equation:

$$
\frac{D \varphi}{D t}=\frac{\partial \varphi}{\partial t}+\frac{d \mathbf{x}}{d t} \cdot \nabla \varphi=0, \quad \varphi(\mathbf{x}, t=0)=\varphi_{0}(\mathbf{x})
$$

where $\varphi_{0}$ is the initial field embedding the interface $\Gamma$ at $t=0, \Gamma_{0} \equiv \Gamma(t=0)$. Introducing the velocity of the interface $\mathbf{V}=d \mathbf{x} / d t$ and its component normal to the interface itself, $\mathcal{V}=\mathbf{V} \cdot \hat{\mathbf{n}}$, where $\hat{\mathbf{n}}=-\nabla \varphi /\|\nabla \varphi\|$, Eq. (1) may be written as follows

$$
\frac{\partial \varphi}{\partial t}=\mathcal{V}(\mathbf{x}, t)\|\nabla \varphi\|
$$

which is the ordinary level set equation, being $\varphi(\mathbf{x}, t)$ the so-called level set function. The interface $\Gamma$ and the burnt region $\Omega$ may be conveniently identified by means of two indicator functions $\mathcal{I}_{\Gamma}, \mathcal{I}_{\Omega}: \mathcal{S} \times[0,+\infty[\rightarrow\{0,1\}$ defined as follows:

$$
\mathcal{I}_{\Gamma}(\mathbf{x}, t)=\left\{\begin{array}{ll}
1 & \text { if } \varphi(\mathbf{x}, t)=\varphi_{*} \\
0 & \text { elsewhere, }
\end{array} \quad I_{\Omega}(\mathbf{x}, t)= \begin{cases}1 & \text { if } \varphi(\mathbf{x}, t) \leqslant \varphi_{*} \\
0 & \text { elsewhere }\end{cases}\right.
$$

The initial topology of the fire is indicated by $\mathcal{I}_{\Gamma_{0}}(\mathbf{x})=\mathcal{I}_{\Gamma}(\mathbf{x}, t=0)$ and $\mathcal{I}_{\Omega_{0}}(\mathbf{x})=$ $I_{\Omega}(\mathbf{x}, t=0)$. In case $\Gamma$ is made up by more than one closed curve, the domain $\Omega$ is not simply connected, resulting in multiple independent burnt areas. When the application to wildfire is considered, the front velocity $\mathcal{V}(\mathbf{x}, t)$ is identified by the RoS, which depends on wind intensity and direction, topography of the terrain, kind of vegetation, etc. Several models for the RoS have been proposed and validated [8, 25-27]; all of them can be straightforwardly implemented in the classical LSM. 
2.3 The randomized level set method

The first step towards the development of the randomized LSM consists in assuming that the motion of the front is affected, in addition to the deterministic RoS, by random effects as turbulence and fire spotting phenomena.

Indexing the realizations by $\omega$, the random trajectory of the particle located at $t=0$ in $\overline{\mathbf{x}}_{0}$ is denoted by $\mathbf{X}^{\omega}\left(t, \overline{\mathbf{x}}_{0}\right)$. Adopting the formalism of statistical mechanics [28], the trajectories are marked out by the one-particle density function $f^{\omega}(\mathbf{x} ; t)=$ $\delta\left(\mathbf{x}-\mathbf{X}^{\omega}\left(t, \overline{\mathbf{x}}_{0}\right)\right)$, where $\delta(\mathbf{x})$ is the Dirac $\delta$-function. Observing that in the deterministic case the level set function $\varphi$, solution of Eq. (2), may be written as

$$
\varphi(\mathbf{x}, t)=\int_{\mathcal{S}} \varphi(\overline{\mathbf{x}}, t) \delta(\mathbf{x}-\overline{\mathbf{x}}) d \overline{\mathbf{x}},
$$

the effects of the random phenomena are included in the model assuming that, in the $\omega$-realization, the level set function $\varphi^{\omega}$ embedding the fireline $\Gamma^{\omega}$ is obtained as a straightforward generalization of Eq. (3) as follows:

$$
\varphi^{\omega}(\mathbf{x}, t)=\int_{\mathcal{S}} \varphi(\overline{\mathbf{x}}, t) \delta\left(\mathbf{x}-\mathbf{X}^{\omega}(t, \overline{\mathbf{x}})\right) d \overline{\mathbf{x}} .
$$

Accordingly, $\mathcal{I}_{\Gamma}$ and $\mathcal{I}_{\Omega}$ are replaced by $\mathcal{I}_{\Gamma^{\omega}}, \mathcal{I}_{\Omega^{\omega}}: \mathcal{S} \times[0,+\infty[\rightarrow\{0,1\}$ defined as:

$$
\begin{aligned}
& \mathcal{I}_{\Gamma^{\omega}}(\mathbf{x}, t)=\int_{\Gamma(t)} \delta\left(\mathbf{x}-\mathbf{X}^{\omega}(t, \overline{\mathbf{x}})\right) d \overline{\mathbf{x}}, \\
& \mathcal{I}_{\Omega^{\omega}}(\mathbf{x}, t)=\int_{\Omega(t)} \delta\left(\mathbf{x}-\mathbf{X}^{\omega}(t, \overline{\mathbf{x}})\right) d \overline{\mathbf{x}},
\end{aligned}
$$

Hence, denoting by $\langle\cdot\rangle$ the ensemble average, the effective indicator of the burnt region, $\varphi_{e}(\mathbf{x}, t): \mathcal{S} \times[0,+\infty[\rightarrow[0,1]$, can be finally introduced:

$$
\varphi_{e}(\mathbf{x}, t)=\left\langle\int_{\Omega(t)} \delta\left(\mathbf{x}-\mathbf{X}^{\omega}(t, \overline{\mathbf{x}})\right) d \overline{\mathbf{x}}\right\rangle=\int_{\Omega(t)} f(\mathbf{x} ; t \mid \overline{\mathbf{x}}) d \overline{\mathbf{x}}=\int_{\mathcal{S}} \mathcal{I}_{\Omega}(\overline{\mathbf{x}}, t) f(\mathbf{x} ; t \mid \overline{\mathbf{x}}) d \overline{\mathbf{x}}
$$

where $f(\mathbf{x} ; t \mid \overline{\mathbf{x}})=\left\langle\delta\left(\mathbf{x}-\mathbf{X}^{\omega}(t, \overline{\mathbf{x}})\right)\right\rangle$ is the PDF of the displacement of the particle [29]. It is worth noting that, since in the deterministic case $\mathbf{X}^{\omega}(t, \overline{\mathbf{x}})=\overline{\mathbf{x}}$ and $f(\mathbf{x} ; t \mid \overline{\mathbf{x}})=$ $\delta(\mathbf{x}-\overline{\mathbf{x}})$, from Eq. (4) it is seen that $\varphi_{e}(\mathbf{x}, t)=I_{\Omega(t)}$.

Applying the Reynolds transport theorem to Eq. (4), the evolution equation of the effective indicator $\varphi_{e}(\mathbf{x}, t)$ reads as [29]:

$$
\frac{\partial \varphi_{e}}{\partial t}=\int_{\Omega(t)} \frac{\partial f}{\partial t} d \overline{\mathbf{x}}+\int_{\Omega(t)} \nabla_{\overline{\mathbf{x}}} \cdot[\mathbf{V}(\overline{\mathbf{x}}, t) f(\mathbf{x} ; t \mid \overline{\mathbf{x}})] d \overline{\mathbf{x}} .
$$

Taking into account that $f(\mathbf{x} ; t \mid \overline{\mathbf{x}})$ satisfies the evolution equation $\partial f / \partial t=\mathcal{E} f$, where $\mathcal{E}=\mathcal{E}(\mathbf{x})$ is a generic evolution operator, Eq. (5) can be written as:

$$
\frac{\partial \varphi_{e}}{\partial t}=\mathcal{E} \varphi_{e}+\int_{\Omega(t)} \nabla_{\overline{\mathbf{x}}} \cdot[\mathbf{V}(\overline{\mathbf{x}}, t) f(\mathbf{x} ; t \mid \overline{\mathbf{x}})] d \overline{\mathbf{x}}
$$


Introducing now the mean front curvature $\kappa=\nabla_{\overline{\mathbf{x}}} \cdot \hat{\mathbf{n}} / 2$ and considering that the front velocity typically depends on the curvature, rather than directly on the position, i.e. $\mathcal{V} \equiv \mathcal{V}(\kappa, t)$, the evolution equation of $\varphi_{e}(\mathbf{x}, t)$ becomes

$$
\frac{\partial \varphi_{e}}{\partial t}=\mathcal{E} \varphi_{e}+\int_{\Omega(t)} \mathbf{V} \cdot \nabla_{\overline{\mathbf{x}}} f d \overline{\mathbf{x}}+\int_{\Omega(t)} f\left\{\frac{\partial \mathcal{V}}{\partial \kappa} \nabla_{\overline{\mathbf{x}}} \kappa \cdot \hat{\mathbf{n}}+2 \mathcal{V}(\kappa, t) \kappa(\overline{\mathbf{x}}, t)\right\} d \overline{\mathbf{x}}
$$

Equation (6) is a reaction-diffusion equation, establishing a connection between reaction-diffusion-based models and models based on the randomized LSM. In particular, the proposed formulation allows to pass from an LSM approach, based on a hyperbolic balance equation (see for example [30]), to a reaction-diffusion model, based on a parabolic balance equation (see for example [31]).

Since the effective indicator $\varphi_{e}$ takes values on the compact interval $[0,1]$, rather than on the discrete set $\{0,1\}$, a criterion to mark the effective burnt region $\Omega_{e}$ is required. A straightforward choice is to mark as burnt the region in which $\varphi_{e}$ exceeds a threshold value $\varphi_{e}^{t h}$, i.e. $\Omega_{e}(\mathbf{x}, t)=\left\{\mathbf{x} \in \mathcal{S} \mid \varphi_{e}(\mathbf{x}, t)>\varphi_{e}^{t h}\right\}$.

\section{Model discussion}

The random trajectory of a particle belonging to the moving front is given by $\mathbf{X}^{\omega}(t, \mathbf{x})=$ $\overline{\mathbf{x}}+\chi^{\omega}+\xi^{\omega}$ where $\overline{\mathbf{x}}_{R o S}$ is the deterministic term computed by means of the chosen RoS model, and $\chi$ and $\xi$ are noises corresponding to turbulence and fire spotting, respectively. These terms are embodied by the PDF $f(\mathbf{x} ; t \mid \overline{\mathbf{x}})$, which is given by the convolution of the PDF associated to the random variable $\left(\overline{\mathbf{x}}_{R o S}+\chi\right)$, in the following indicated as $G$, and the one associated to $\xi$, in the following indicated as $q$.

In the present model, the fire spotting noise $\xi$ is aligned with the mean wind direction $\hat{\mathbf{n}}_{U}$, i.e. $\xi^{\omega}=\ell^{\omega} \hat{\mathbf{n}}_{U}$, being $\ell$ the landing distance of the firebrands from the front. Moreover, turbulent noise $\chi$ is a zero-mean noise, i.e. $\langle\chi\rangle=0$, while fire spotting noise $\xi$ has a positive mean value, i.e. $\langle\ell\rangle>0$, being the wind velocity the same in all realizations. Therefore, the average position in the leeward sector is $\langle\mathbf{X}(t, \overline{\mathbf{x}})\rangle=\overline{\mathbf{x}}+\langle\ell\rangle \hat{\mathbf{n}}_{U}$, while in the windward sector is $\langle\mathbf{X}(t, \overline{\mathbf{x}})\rangle=\overline{\mathbf{x}}$. It should also be considered that fire spotting is to be taken into account only in the leeward part of the front, being fire spotting an intrinsically downwind phenomenon. Therefore,

$$
f(\mathbf{x} ; t \mid \overline{\mathbf{x}})= \begin{cases}\int_{0}^{\infty} G\left(\mathbf{x}-\overline{\mathbf{x}}-\ell \hat{\mathbf{n}}_{U} ; t\right) q(\ell ; t) d \ell & \text { where } \hat{\mathbf{n}} \cdot \hat{\mathbf{n}}_{U} \geqslant 0, \\ G(\mathbf{x}-\overline{\mathbf{x}} ; t) & \text { otherwise }\end{cases}
$$

Concerning turbulent phenomena, the most simple model is the Gaussian one that, in the isotropic case, provides the following PDF:

$$
G(\mathbf{x}-\overline{\mathbf{x}} ; t)=\frac{1}{2 \pi \sigma^{2}(t)} \exp \left(-\frac{(x-\bar{x})^{2}+(y-\bar{y})^{2}}{2 \sigma^{2}(t)}\right),
$$

where $\mathbf{x} \equiv(x, y), \overline{\mathbf{x}} \equiv(\bar{x}, \bar{y})$, and $\sigma^{2}(t)=\left\langle(\mathbf{x}-\overline{\mathbf{x}})^{2}\right\rangle / 2=2 \mathcal{D} t$ is the particle displacement variance, being $\mathcal{D}$ the turbulent diffusion coefficient. 
For what concerns the fire spotting phenomenon, it has been argued [18] that the landing distance of the firebrands follows a log-normal distribution:

$$
q(\ell ; t)=\frac{1}{\sqrt{2 \pi} s(t) \ell} \exp \left\{-\frac{(\ln \ell-\mu(t))^{2}}{2 s(t)^{2}}\right\},
$$

where $\mu(t)=\langle\ln \ell\rangle$ and $s(t)=\left\langle(\ln \ell-\mu(t))^{2}\right\rangle$ are, respectively, the mean and the standard deviation of $\ln \ell$.

It should be emphasized here that, being $\langle\chi\rangle=0$, the noise due to turbulent transport does not affect the average position of the front, $\langle\mathbf{X}(t, \overline{\mathbf{x}})\rangle$. The same cannot be said when fire spotting is also considered. In fact, being $\langle\ell(t)\rangle>0$, in the leeward front sector, the advancement of the fire is enhanced by fire spotting, $\langle\mathbf{X}(t, \overline{\mathbf{x}})\rangle=$ $\overline{\mathbf{x}}(t)+\langle\chi\rangle+\langle\xi\rangle=\overline{\mathbf{x}}(t)+\langle\ell(t)\rangle \hat{\mathbf{n}}_{U}$, being $\hat{\mathbf{n}}_{U}$ the unit vector pointing outward of the burned domain. As a consequence, when the fire spotting is included, the velocity of the mean front in the leeward sector is incremented by the contribution due to the deterministic RoS, $\mathbf{V}_{R o S}$,

$\mathbf{V}(\overline{\mathbf{x}}, t)=\frac{d\left(\overline{\mathbf{x}}_{R o S}+\langle\ell\rangle \hat{\mathbf{n}}_{U}\right)}{d t}=\mathcal{V}_{R o S}(\overline{\mathbf{x}}, t) \hat{\mathbf{n}}+\frac{d\langle\ell\rangle}{d t} \hat{\mathbf{n}}_{U}+\langle\ell\rangle \frac{d \hat{\mathbf{n}}_{U}}{d t}=\mathbf{V}_{R o S}(\overline{\mathbf{x}}, t)+\mathbf{V}_{\ell}(\overline{\mathbf{x}}, t)$, and the level set equation in the leeward sector must be modified as follows in order to incorporate the effect of the fire spotting:

$$
\frac{\partial \varphi}{\partial t}=\left(\mathcal{V}_{R o S}+\mathbf{V}_{\ell} \cdot \hat{\mathbf{n}}\right)\|\nabla \varphi\| .
$$

Another important result of the proposed approach is the possibility to manage real world cases in which fire overcomes a zone without fuel, like roads or artificial firebreak (this feature of the model was reported also in the case in which only turbulence was taken into account $[10,11])$.

\section{Numerical results}

The model discussed in the previous section has been implemented in a general purpose LSM-based parallel code thoroughly described elsewhere $[32,33]$.

Since the aim of the present paper is to investigate the potentialities of the model, rather than simulate a wildfire under realistic conditions, the results presented here are restricted to oversimplified cases in order to highlight the role played by the random phenomena and the effects of fire-breaks in the region affected by the fire.

Details concerning the simulation set-up and the environmental input data are not provided here for the sake of brevity, being the same as those previously detailed by other authors $[16,18,20]$. It is only mentioned here that the deterministic rate of spread is computed on the basis of the Byram formula [34] suitably modified in order to include the wind and slope effects as prescribed in [13]. The direction of the mean wind is $90^{\circ}$ clockwise from North (i.e. from West to East) for the first 90 minutes, than it changes gradually rotating clockwise of $1 \% / 4$ minutes.

With the purpose of pointing out the main features of the model and the effects of fire-breaks like roads, three cases have been selected for discussion among the pool of 
new available numerical results. These cases are characterized by values of the wind velocity ranging from 6.7 to $17.88 \mathrm{~m} / \mathrm{s}$ and values of the fire intensity $I$ ranging from 5000 to $20000 \mathrm{~kW} / \mathrm{m}\left(U_{t}=6.7\right.$ and $I=5000 \mathrm{~kW} / \mathrm{m}$ for case $\mathcal{A}$, shown in Figure 1 ; $U_{t}=6.7$ and $I=10000 \mathrm{~kW} / \mathrm{m}$ for case $\mathcal{B}$, shown in Figure 2; $U_{t}=17.88$ and $I=$ $20000 \mathrm{~kW} / \mathrm{m}$ for case $C$, shown in Figure 3). In all the cases, numerical simulations have been performed assuming a deterministic front propagation, as well as including random effects due only to turbulence with and without fire spotting.

In each case, the evolution of the front propagating in a terrain with no fire-breaks are shown on the left, and the corresponding evolution in presence of fire-breaks (grey regions, representing roads or similar fuel-free obstacles) is shown on the right. For both cases, the results obtained by adopting three different models are shown in the figures: the deterministic model, in which the front is tracked by means of the classical level set method (top raw of each figure); the model in which the front is tracked by means of the level set method including only the turbulence effects (middle raw), and the full-featured model presented in the previous section in which the fire spotting phenomenon is also included (bottom raw).

As a general rule, comparing the results obtained in the randomized approach to those obtained in the deterministic framework, it is possible to state that, as expected, the fire front propagates faster when turbulence effects are taken into account.

In addition, the numerical results strongly suggest the importance of the fire spotting phenomenon as a mechanism enhancing the front propagation: This is particularly evident in the cases in which the fire propagates in a region in which fire-breaks are present. In this situation, the modelling results strikingly point out how the fire spotting phenomenon may be crucial in making the fire overcoming the fire-breaks faster than when adopting a model including only turbulence effects. As it has been previously shown $[10,11]$, the turbulence itself can be responsible for the spreading of the wildfire across fire-breaks; but it appears clearly, when comparing the results of Figures 1(d), 2(d) and 3(d) to those of Figures 1(f), 2(f) and 3(f), that the fire spotting phenomenon is capable of remarkably enhancing this capability of the wildfire.

Acknowledgements This research was supported by GNFM/INdAM Young Researchers Project 2015 'An Eulerian/Lagrangian model for combustion fronts', by MINECO under Grant MTM2013-40824-P, by Bizkaia Talent and European Commission through COFUND programme under Grant AYD-000-226, by the Basque Government through the BERC 2014-2017 program, and by the Spanish Ministry of Economy and Competitiveness MINECO: BCAM Severo Ochoa accreditation SEV-2013-0323.

\section{References}

1. Sullivan, A.: A review of wildland fire spread modelling, 1990-present, 1: Physical and quasi-physical models. Int. J. Wildland Fire 18, 349-368 (2009)

2. Sullivan, A.: A review of wildland fire spread modelling, 1990-present, 2: Empirical and quasiempirical models. Int. J. Wildland Fire 18, 369-386 (2009)

3. Sullivan, A.: Wildland surface fire spread modelling, 1990-2007. 3: Simulation and mathematical analogue models. Int. J. Wildland Fire 18, 387-403 (2009)

4. Asensio, M. I., Ferragut, L.: On a wildland fire model with radiation. Int. J. Numer. Meth. Engng. 54, 137-157 (2002)

5. Mandel, J., Bennethum, L. S., Beezley, J. D., Coen, J. L., Douglas, C. C., Kim, M., Vodacek, A.: A wildland fire model with data assimilation. Math. Comput. Simulat. 79, 584-606 (2008) 

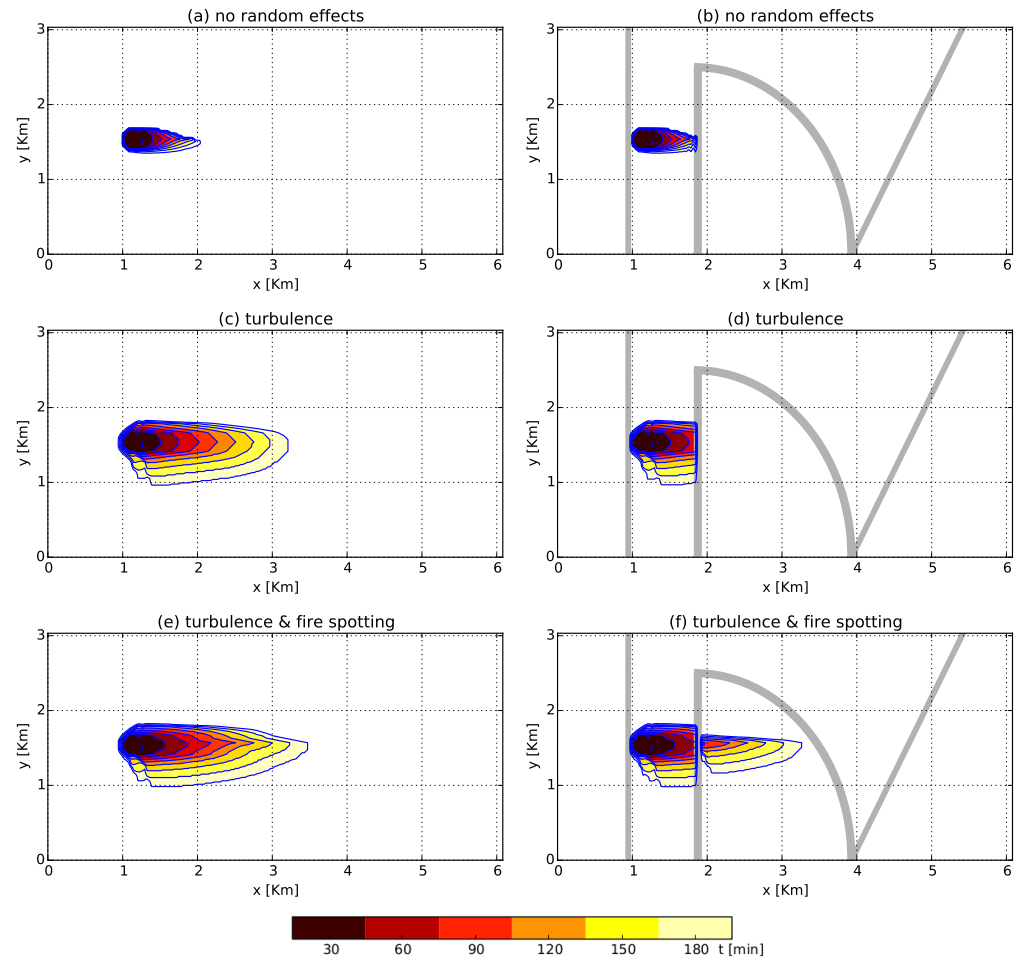

Fig. 1 Evolution of the front in case $\mathcal{A}\left(U_{t}=6.70 \mathrm{~m} / \mathrm{s}, I=5000 \mathrm{~kW} / \mathrm{m}\right)$, in absence (left) and presence (right) of fire-breaks (grey regions). The results are obtained by means of the classical LSM (top row) and by the randomized LSM including turbulence without (middle raw) and with (bottom row) fire spotting. (The darkest/lightest region represent the extension of the wildfire after 30/180 minutes (intermediate color regions are snapshots taken each 20 minutes).

6. Babak, P., Bourlioux, A., Hillen, T.: The effect of wind on the propagation of an idealized forest fire SIAM J. Appl. Math. 70, 1364-1388 (2009)

7. Sethian, J. A., Smereka, P.: Level set methods for fluid interfaces. Ann. Rev. Fluid Mech. 35, 341-372 (2003)

8. Mallet, V., Keyes, D. E., Fendell, F. E.: Modeling wildland fire propagation with level set methods Comput. Math. Appl. 57, 1089-1101 (2009)

9. Rehm, R. G., McDermott, R. J.: Fire-Front Propagation Using the Level Set Method. Tech. Note 1611, Natl. Inst. Stand. Technol. (2009)

10. Pagnini, G., Massidda, L.: The randomized level set method to model turbulence effects in wildland fire propagation. In: Proc. of the Int. Conf. on Fire Behaviour and Risk (ICFBR2011), 126-131 (2012)

11. Pagnini, G., Massidda, L.: Modelling turbulence effects in wildland fire propagation by the randomized level set method. CRS4 Technical Report 2012/PM12a, July 2012. Revised Version August 2014. http://publications.crs4.it/pubdocs/2012/PM12a/pagnini_ massidda-levelset.pdf (2014)

12. Filippi, J. B., Bosseur, F., Mari, C., Lac, C., Moigne, P. L., Cuenot, B., Veynante, D., Cariolle, D., Balbi, J. H.: Coupled atmosphere-wildland fire modelling. J. Adv. Model. Earth Syst. 1 (2009)

13. Mandel, J., Beezley, J. D., Kochanski, A. K.: Coupled atmosphere-wildland fire modeling with WRF 3.3 and SFIRE 2011. Geosci. Model Dev. 4, 591-610 (2011)

14. Potter, B. E.: Atmospheric interactions with wildland fire behaviour - I. Basic surface interactions, vertical profiles and synoptic structures. Int. J. Wildland Fire 21, 779-801 (2012) 

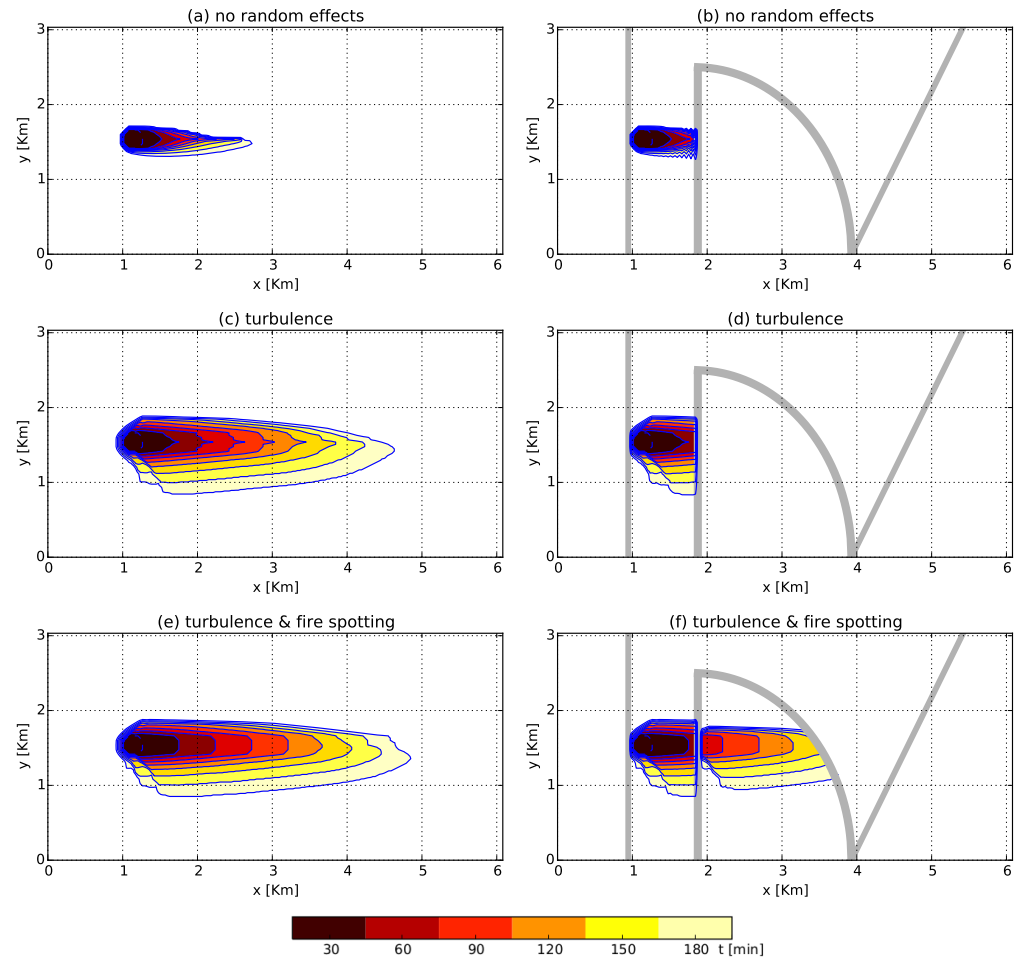

Fig. 2 Evolution of the front in case $\mathcal{B}\left(U_{t}=6.70 \mathrm{~m} / \mathrm{s}, I=10000 \mathrm{~kW} / \mathrm{m}\right)$, in absence (left) and presence (right) of fire-breaks (grey regions). The results are obtained by means of the classical LSM (top row) and by the randomized LSM including turbulence without (middle raw) and with (bottom row) fire spotting. (The darkest/lightest region represent the extension of the wildfire after 30/180 minutes (intermediate color regions are snapshots taken each 20 minutes).

15. Potter, B. E.: Atmospheric interactions with wildland fire behaviour - II. Plume and vortex dynamics. Int. J. Wildland Fire 21, 802-817 (2012)

16. Sardoy, N., Consalvi, J. L., Porterie, B., Fernandez-Pello, A. C.: Modeling transport and combustion of firebrands from burning trees. Combust. Flame 150, 151-169 (2007)

17. Perryman, H. A.: A Mathematical Model of Spot Fires and their Management Implications. Master's thesis, Humboldt State University, Arcata, CA (2009)

18. Sardoy, N., Consalvi, J. L., Kaiss, A., Fernandez-Pello, A. C., Porterie, B.: Numerical study of groundlevel distribution of firebrands generated by line fires. Combust. Flame 154, 478-488 (2008)

19. Boychuk, D., Braun, W. J., Kulperger, R. J., Krougly, Z. L., Stanford, D. A.: A stochastic forest fire growth model. Environ. Ecol. Stat. 16, 133-151 (2009)

20. Pagnini, G., Mentrelli, A.: Modelling wildland fire propagation by tracking random fronts. Nat. Hazards Earth Syst. Sci. 14, 2249-2263 (2014)

21. Kaur, I., Mentrelli, A., Bosseur, F., Filippi, J.-B., Pagnini, G.: Wildland Fire Propagation Modelling: A Novel Approach Reconciling Models Based on Moving Interface Methods and on ReactionDiffusion Equations. In: Proceedings of the International Conference Applications of Mathematics 2015 (Prague, CZ; November 18-21, 2015); Eds. J. Brandts et al.; Institute of Mathematics AS CR, Prague, 85-99 (2015)

22. Kaur, I., Mentrelli, A., Bosseur, F., Filippi J.-B., Pagnini, G.: Turbulence and fire-spotting effects into wild-land fire simulators. arXiv:1601.06272

23. Montenegro, R., Plaza, A., Ferragut, L., Asensio, M. I.: Application of a nonlinear evolution model to fire propagation. Nonlinear Anal. Theory Methods Appl. 30, 2873-2882 (1997) 

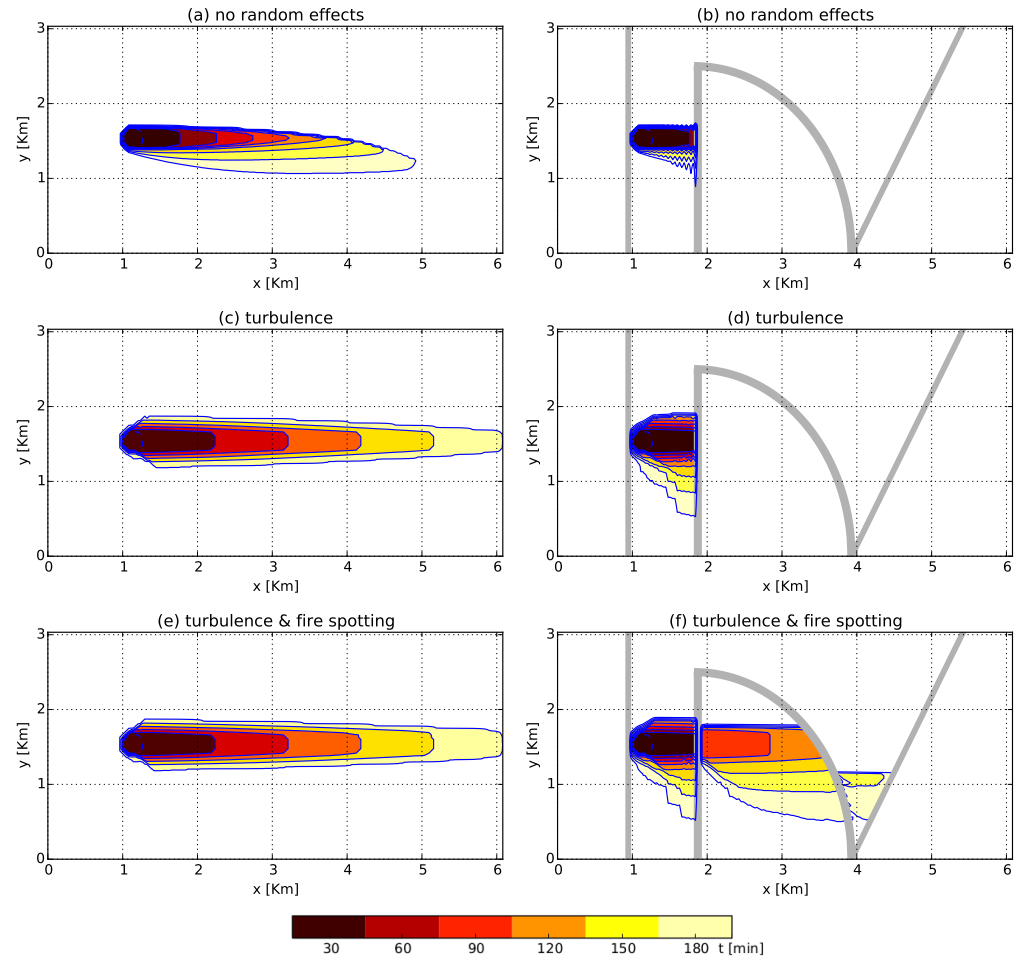

Fig. 3 Evolution of the front in case $C\left(U_{t}=17.88 \mathrm{~m} / \mathrm{s}, I=20000 \mathrm{~kW} / \mathrm{m}\right)$, in absence (left) and presence (right) of fire-breaks (grey regions). The results are obtained by means of the classical LSM (top row) and by the randomized LSM including turbulence without (middle raw) and with (bottom row) fire spotting. (The darkest/lightest region represent the extension of the wildfire after 30/180 minutes (intermediate color regions are snapshots taken each 20 minutes).

24. Serón, F. J., Gutiérrez, D., Magallón, J., Ferragut, L., Asensio, M. I.: The evolution of a wildland forest fire front. Visual Comput. 21, 152-169 (2005)

25. Rothermel, R. C.: A Mathematical Model for Predicting Fire Spread in Wildland Fires. Tech. Rep. Research Paper INT-115, USDA Forest Service, Intermountain Forest and Range Experiment Station, Ogden, Utah 84401 (1972)

26. Finney, M.: Calculation of fire spread rates across random landscapes. Int. J. Wildland Fire 12, 167174 (2003)

27. Balbi, J. H., Morandini, F., Silvani, X., Filippi, J. B., Rinieri, F.: A physical model for wildland fires. Combust. Flame 156, 2217-2230 (2009)

28. Klimontovich, Y. L.: Nonlinear Brownian motion. Phys.-Uspekh. 37, 737-767 (1994)

29. Pagnini, G., Bonomi, E.: Lagrangian formulation of turbulent premixed combustion. Phys. Rev. Lett. 107, $044503(2011)$

30. Dafermos, C. M.: Continuous solutions for balance laws. Ric. Mat. 55, 79-91 (2006)

31. De Angelis, M., Renno, P.: Existence, uniqueness and a priori estimates for a nonlinear integrodifferential equation. Ric. Mat. 57, 95-109 (2008)

32. Mentrelli, A., Pagnini, G.: Random front propagation in fractional diffusive systems. Commun. Appl. Ind. Math. 6 (2), e-504 (2014)

33. Mentrelli, A., Pagnini, G.: Front Propagation in Anomalous Diffusive Media Governed by TimeFractional Diffusion. J. Comput. Phys. 293, 427-441 (2015)

34. Byram, G. M.: Combustion of forest fuels. In: Forest Fire: Control and Use (Ed. K. P. Davis), 61-89, McGraw Hill, New York (1959) 